\title{
SISTEMAS DE INFORMACIÓN PARA LA GESTIÓN DE RIESGOS EN LAS TRIPULACIONES DE VUELO
}

\section{INFORMATION SYSTEMS FOR RISK MANAGEMENT IN FLIGHT CREWS}

\author{
Rosero-Pineda Roberto ${ }^{1 *}$; Acevedo-Almonacid Héctor ${ }^{2}$ \\ ${ }^{1}$ Universidad Técnica Federico Santa María, USM. Guayaquil, Ecuador. \\ ${ }^{2}$ Docente de la Universidad Técnica Federico Santa María, USM. Guayaquil, Ecuador.
}

*Correo: Roberto-rosero-pineda@gmail.com

\begin{abstract}
Resumen
Los accidentes aéreos provocan grandes pérdidas materiales y de personas, recursos que son vitales e importantes para toda organización. El presente artículo tiene como objetivo establecer una revisión de los sistemas de información para la gestión de la seguridad y prevención de accidentes en los procesos de aviación. Se propone que la efectividad de la evaluación del nivel de riesgo al que se exponen los pilotos aéreos demanda recoger, almacenar, procesar y distribuir a tiempo información relevante para diagnosticar y detectar la restricción a las tripulaciones de vuelo antes del cumplimiento de la misión, por lo tanto, el sistema de información para la gestión (SI) engloba todas estas tareas, cometidas y recursos humanos que están orientados a proporcionar las necesidades de información del sistema de decisión. Finalmente, se considera que el hombre, máquina y entorno son componentes de un solo sistema, y por tanto debe considerarse que las causas de un accidente son deficiencias, fallas, errores o discrepancias que se producen durante la interacción e interrelación, de las distintas variables de cada componente.
\end{abstract}

Palabras clave: gestión, prevención, riesgos, accidentes, tecnología de información.

\begin{abstract}
Plane crashes cause great loss of material and people, resources that are vital and important to any organization. This article aims to establish a review of information systems for the management of safety and accident prevention in aviation processes. It is proposed that the effectiveness of the evaluation of the level of risk to which air pilots are exposed demands to collect, store, process and distribute relevant information in time to diagnose and detect the restriction to flight crews prior to the completion of the mission, by Therefore, the management information system (IS) encompasses all these tasks, tasks and human resources that are aimed at providing the information needs of the decision system. Finally, man, machine and environment are considered to be components of a single system, and therefore it must be considered that the causes of an accident are deficiencies, failures, errors or discrepancies that occur during the interaction and interrelation of the different variables. of each component.
\end{abstract}

Keywords: management, prevention, risks, accidents, information technology.

Información del manuscrito:

Fecha de recepción: 15 de marzo de 2017

Fecha de aceptación: 06 de junio de 2017

Fecha de publicación: 10 de julio de 2017 


\section{Introducción}

Los accidentes aéreos provocan grandes pérdidas materiales y de personas, recursos que son vitales e importantes para toda organización y la sociedad en general (Sánchez \& Lina, 2008).

La prevención involucra un conjunto de actividades destinadas a evitar o minimizar los riesgos potenciales que contribuyen a la concurrencia de un accidente y para ello se debe considerar la estrecha relación que existe entre el hombre, la máquina y el medio ambiente. Un análisis adecuado de estos tres factores permitirá identificar esos riesgos y tomar medidas correctivas eficaces, reales y objetivas; considerando que la gran mayoría de los accidentes aéreos son el resultado de una decisión errada, un descuido o negligencia, es decir su origen es fundamentalmente humano (DíazCabrera et al., 2008).

Por tanto, la obtención oportuna de datos fiables para conocer las posibilidades de riesgos que representan las tripulaciones de vuelo antes de cumplir una misión es de carácter fundamental, esto en aras de disminuir la ocurrencia de incidentes 0 accidentes aéreos.
El presente artículo tiene como objetivo establecer una revisión de los sistemas de información para la gestión de la seguridad y prevención de accidentes en los procesos de aviación.

\section{Soluciones de gestión de sistemas de información y tecnologías de información}

Los Sistemas de Información (SI) y las Tecnologías de Información (TI) han cambiado la forma en que operan las organizaciones actuales. A través de su uso se logran importantes mejoras, pues automatizan los procesos, proveen información necesaria para la toma de decisiones y alcanzan ventajas competitivas.

La efectividad para la evaluación del nivel de riesgo a los pilotos aéreos demanda recoger, almacenar, procesar y distribuir a tiempo información relevante para diagnosticar y detectar la restricción a las tripulaciones de vuelo antes del cumplimiento de la misión, con la finalidad que el Oficial de Operaciones pueda decidir y actuar de la forma más eficaz posible en la toma de decisiones (Lema-Pazmiño, 2017). El sistema de información para la gestión (SI) engloba todas 
estas tareas, cometidas y recursos

humanos que están orientados a proporcionar las necesidades de

información del sistema de decisión
(Pechuán, 1997). La figura 1 muestra los componentes del SI.

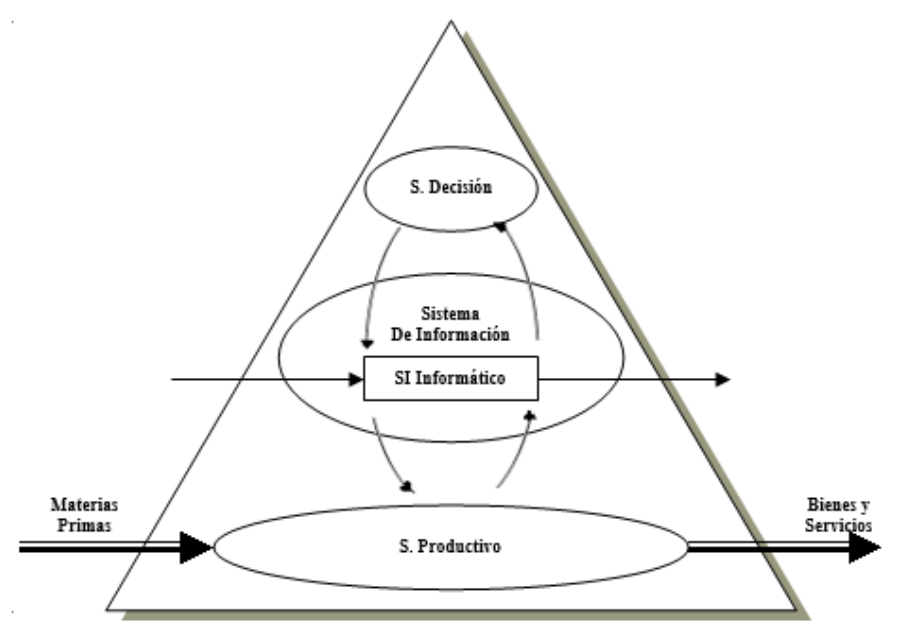

Figura 1. Sistema de información en la Organización

Así, el SI está totalmente imbricado en la organización a la que sirve, de lo que se desprende que su planificación, administración, diseño e implementación deben asociarse estrechamente con las tareas de planificación, administración, diseño e implementación de la misma organización. Además de las funciones intrínsecas de todo SI, vistas en la figura 2, conviene enumerar los propósitos organizativos del SI indicando así su para qué y su por qué:

- Las organizaciones necesitan que la información relevante fluya para así poder planificar, controlar y coordinar sus actividades operativas de la manera más eficiente posible.

- Las organizaciones precisan adquirir otras informaciones relevantes para su funcionamiento, organizadas, almacenadas, procesadas y distribuidas a tiempo, para que las personas puedan decidir y actuar de la forma más eficaz posible (Laudon \& Rodríguez, 1996).

- Tanto la eficiencia en las operaciones como la eficacia en las decisiones están supeditadas al mantenimiento de la máxima coherencia posible con la misión, los objetivos y las estrategias de 
la organización; en otras palabras, para así dirigir de forma efectiva la organización.

- A su vez, todo lo anterior mejora cuando existe una adecuada comunicación y ambiente cooperativo de trabajo entre las personas de la organización adquiriendo así algo que podemos denominar un buen nivel de emotividad organizativa.

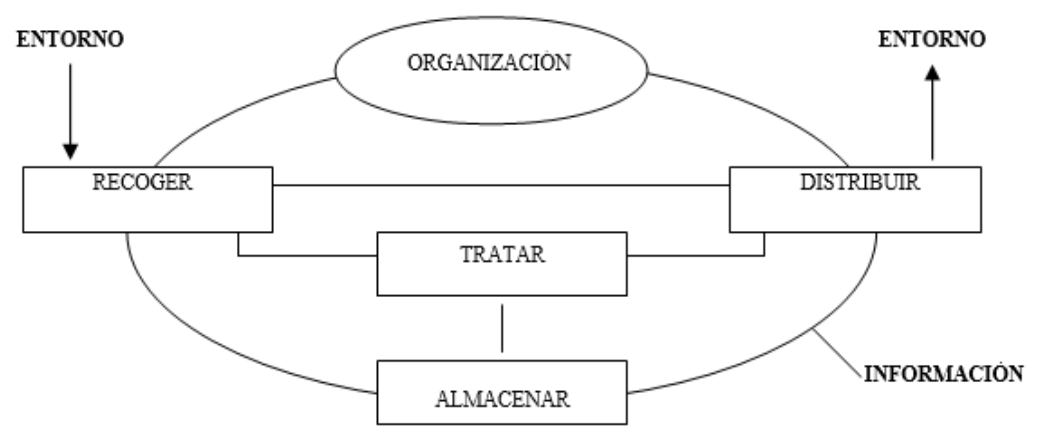

Figura 2. Funciones intrínsecas del Sistema de Información

Podemos inducir que los componentes más elementales que conforman al Sistema de Información son conjuntos de información y procesos de información. En ambos casos se trata de conceptos abstractos que se pueden implementar de muchas formas en el mundo físico, pero que giran alrededor del concepto de información (Pechuán, 1997). En otras palabras, a la información se la puede considerar como la materia prima de todo sistema de información (Fernández-Alarcón, 2006).
3. Soluciones organizacionales para la evaluación de riesgos en las tripulaciones de vuelo

La administración de riesgos es reconocida como una parte integral de las buenas prácticas gerenciales. Este es un proceso iterativo que consta de pasos, los cuales cuando son ejecutados en secuencia, posibilitan una mejora continua en el proceso de toma de decisiones.

La formación de pilotos listos para el combate, significa someter a las personas a unas exigencias altas para cumplir con la función requerida en un tiempo determinado y con una calidad establecida, disminuyendo la 
posibilidad de fallo, haciendo cada vez más necesario el análisis, evaluación y cuantificación del riesgo de "error humano", con el fin de lograr eliminarlo o minimizarlo, para que este no interfiera en la producción de vuelos ni en la seguridad de estos (Alvernia, 2008). A pesar de que la fisonomía del ser humano indica que por naturaleza éste no es apto para volar; para ser piloto no solo se necesita tener el deseo sino contar con unos rasgos básicos de personalidad orientados a mantener un adecuado manejo del stress, seguridad en la toma de decisiones, un alto sentido del orientación y percepción y tener aptitudes psicomotrices muy bien fundamentadas; puesto que las estadísticas demuestran que un gran porcentaje de los problemas de accidentes aéreos son fallas humanas, producto de una deficiente doctrina de prevención (Rubio, 2010).

Partiendo de la nueva percepción del error humano que dice:

- El error humano es síntoma de problemas profundos dentro de un sistema.
- Para explicar la falla, no hay que tratar de averiguar dónde se equivocaron las personas.

- Lo que se debe investigar es cómo las apreciaciones y las acciones de las personas tenían sentido en el momento, dadas las circunstancias que las rodeaban.

Podemos plantearnos preguntas como:

¿Cuáles son las ventajas de la nueva percepción y su aplicación en el mundo de la gestión de la seguridad?

- Se trata de los factores subyacentes en lugar de los síntomas.

- Se enfocan las cuestiones desde una perspectiva sistémica 0 multidimensional.

- Se aplican métodos científicamente válidos para comprender el funcionamiento del sistema.

¿Cuáles son las razones para ocuparse de los factores humanos?

Los humanos son:

- El componente más flexible, adaptable, y valioso del sistema de la aviación. 
- El más vulnerable a factores que pueden influir negativamente en su comportamiento.

- La mayoría de los accidentes se han atribuido al "error Humano".

Considerando la estrecha relación que existe entre el hombre, la máquina y el entorno como componentes de un todo, las causas son deficiencias, fallas, errores 0 discrepancias que se producen durante la interacción e interrelación, de las distintas variables de cada componente.

Las fallas pueden presentarse en todos los niveles desde la planificación, dirección, ejecución, supervisión y control, hasta la evaluación de cualquier actividad aeronáutica; por lo tanto, la Institución debe optar por un enfoque amplio organizacional que permita identificar, de que manera tales variables pudieron haberse integrado e interactuado entre Hombre-Hombre, Hombre-Máquina, Hombre-Medio ambiente o entorno, para dar lugar al accidente.

\subsection{Prevención de accidentes}

La prevención de accidentes solo se conseguirá con la participación activa de todos los miembros de la
Organización en las tareas de detección e identificación de cualquier situación de peligro que pudiera ocasionar un accidente (Rubio, 2010).

La implementación puesta en ejecución y control del cumplimiento de los programas de prevención de accidentes es responsabilidad del comandante, mismo que debe proporcionar el estímulo necesario para asegurar el éxito de un programa de seguridad.

Las actividades de prevención exigen una organización que permita identificar en forma oportuna las áreas de mayor incidencia de peligros potenciales y aplicar las medidas correctivas necesarias; para ello, se debe contar con personal altamente capacitado en materia de prevención e investigación, procedimientos eficaces y ágiles, infraestructura acorde a su naturaleza y recursos económicos suficientes (Alvernia, 2008).

\section{Conclusiones}

El hombre, máquina y entorno son componentes de un solo sistema, y por tanto debe considerarse que las causas de un accidente son 
deficiencias, fallas, errores 0 discrepancias que se producen durante la interacción e interrelación, de las distintas variables de cada componente. Considerando que estas fallas pueden presentarse en todos los niveles, desde la planificación, dirección, ejecución, supervisión y control, hasta la evaluación de cualquier actividad aeronáutica 0 terrestre, se debe adoptar un enfoque amplio que permita identificar, de que manera tales variables pudieron haberse integrado e interactuado para dar lugar al accidente.

La evaluación del nivel de riesgos que representan las tripulaciones de vuelo, demanda de mucha disciplina por parte de los responsables; el control, el cumplimiento debe ser muy riguroso a todo nivel, por consiguiente, debe garantizarse en el apoyo de la alta dirección y en la participación de todos los involucrados en el proceso para mantener la seguridad aérea.

\section{Bibliografía}

Alvernia, E. L. (2008). La seguridad en la aviación depende de los factores humanos. Ciencia $y$ poder aéreo, 3(1), 37-39.
Díaz-Cabrera, D., Isla-Díaz, R., Rolo-González, G., VillegasVelásquez, O., RamosSapena, Y., \& HernándezFernaud, E. (2008). La salud y la seguridad organizacional desde una perspectiva integradora. Papeles del psicólogo, 29(1), 83-91.

Fernández-Alarcón, V. (2006). Desarrollo de sistemas de información: una metodología basada en el modelado.

Laudon, K. C., \& RodríguezRodríguez, J. (1996). Administración de los sistemas de información: organización y tecnología.

Lema-Pazmiño, G. X. (2017). Diseño de las buenas prácticas del sistema de gestión de la seguridad de la información basado en normas ISO 27001 para la dirección general de Aviación Civil (Bachelor's thesis, Quito: Universidad de las Américas, 2017).

Pechuán, I. G. (1997). Sistemas y Tecnologías de la Información para la gestión. McGraw-Hill.

Rubio, L. M. S. (2010). El estudio del factor humano en accidentes de aviación. Pensamiento Psicológico, 7(14).

Sánchez, L. M., \& Lina, M. (2008). Medicina aeroespacial y factores humanos en aviación. La importancia de una aproximación transdisciplinaria a la salud. Revista Med, 16(2), 249-260. 